\title{
DIRECT SUM THEOREM FOR HAAR MEASURES
}

\author{
BY \\ W. AMBROSE
}

In this paper we prove a result needed in the preceding paper, publishing it separately because it is so different from most of the material of that paper. This result is an extension of a formula of Weil-or rather an extension of a special case of Weil's formula [5, pp. 42-45]( $\left.{ }^{1}\right)$.

Let $G$ be a locally compact topological group, $G^{\prime}$ a closed invariant subgroup, and $G_{1}$ the quotient group $G / G^{\prime}$. Then each of $G, G^{\prime}$ and $G_{1}$ has a left invariant Haar measure and we denote these three measures by $m, m^{\prime}$ and $m_{1}$, respectively. The Weil formula asserts, in this case, that if $f(x)$ is any continuous function on $G$ which vanishes outside some compact set then

$$
\int_{G} f(x) d m=\int_{G_{1}}\left\{\int_{G^{\prime}} f\left(x x^{\prime}\right) d m^{\prime}\right\} d m_{1} .
$$

The object of this paper is to extend this formula and formalize this relation under the notion of a "direct sum" of measure spaces, which was introduced and used by P. R. Halmos $[1,2]$.

Actually Weil proves a more general formula than (1) because he allows $G^{\prime}$ to be a non-invariant closed subgroup of $G$, then works with $G_{1}$, the space of left cosets, proves the existence of a "relatively invariant" measure on $G_{1}$, and then proves (1) in that case [5, pp. 42-45]. In particularly he proves the existence of a measure, which exists even when Haar measure doesn't, on the quotient space $G_{1}$, so his contribution is an existence theorem for measures as well as formula (1). We could extend this more general formula of Weil but that would be more complicated, so we treat only the case needed in the preceding paper.

We use freely the notation, terminology and definitions of $\$ 1$ of the preceding paper.

Definition (2). Let $X_{1}$ be a measure space with a measure $m_{1}$, and suppose that for each $x \in X_{1}$ we have a measure space $Y_{x}$ with measure $m_{x}$. Let $X$ be the space of ordered pairs $(x, y)$ where $y \in Y_{x}$ and suppose that $X$ is a measure space in its own right with measure $m . X$ is the direct sum of the measure spaces $Y_{x}$ by the measure space $X_{1}$ (or equivalently we say $m$ is the direct sum of the measures $m_{x}$ by the measure $m_{1}$ ) if for every $m$-measurable set $E$ the set $E_{x}$ of all $y$ such that $(x, y) \in E$ is $m_{x}$-measurable except possibly for a set

Presented to the Society, November 2, 1946; received by the editors February 25, 1946.

(1) Numbers in brackets refer to the bibliography at the end of the paper.

(2) This definition is given in [1] and [2]. 
of $x$ 's of $m_{1}$-measure 0 and

$$
m E=\int_{X_{1}} m_{x}\left(E_{x}\right) d m_{1}
$$

including the assertion that $m_{x}\left(E_{x}\right)$ is an $m_{1}$-integrable function. We denote this relation by $m=\int m_{x} d m_{1}$.

We remark that two different measures on $X$ could both be the direct sum of the $m_{x}$ 's by $m_{1}$, in case their measurable sets were not the same, but that two such measures would have to agree on any set that was measurable with respect to both.

The main theorem of this paper says that, in the situation described in the second paragraph of this paper, $m$-measure on $G$ is the direct sum of $m^{\prime}$-measure on the cosets of $G^{\prime}$ by $m_{1}$-measure on $G_{1}$. By " $m^{\prime}$-measure on a coset" we mean the "transplanted" measure on the coset $x G^{\prime}$ defined by the assertion that the mapping $y^{\prime} \rightarrow x y^{\prime}$ of $G^{\prime}$ onto $x G^{\prime}$ shall be measure preserving. We have mentioned in $\S 1$ of the preceding paper that this measure on $x G^{\prime}$ is independent of the $x$ used in representing the coset. Now we state the main theorem precisely.

THEOREM 1. Let $G$ be a locally compact topological group, $G^{\prime}$ a closed invariant subgroup of $G$ (considered in the topology induced from $G$ ) and $G_{1}$ the corresponding quotient group. Let the left invariant Haar measures of $G, G^{\prime}$ and $G_{1}$ be denoted respectively by $m, m^{\prime}$ and $m_{1}$ and also use the notation $m^{\prime}$ for the transplanted measure on the cosets of $G^{\prime}$ in $G$. Then m-measure is, to within a constant factor, the direct sum of $m^{\prime}$-measure on the cosets by $m_{1}$-measure on $G_{1}$, that is, $m=k \int m^{\prime} d m_{1}$.

It is clear that this theorem, by asserting that (2) holds with these measures, says the same thing about the $m$-measurable sets that (1) says about the continuous functions which vanish outside a compact set. However (2) almost immediately implies that (1) holds for all integrable $f$; one has only to note that if one measure space is a direct sum of others then its product with the real line is the direct sum of their products with the real line. Since an integral is just a measure in a product space this fact yields (1) for all integrable $f$.

Our proof of Theorem 1 goes via the following route: We define $m^{*}$ on $G$ by $m^{*}=\int m^{\prime} d m_{1}$, that is, we consider the sets $E$ such that $E_{x}=E \cap x G^{\prime}$ is $m^{\prime}$-measurable, and $m^{\prime}\left(E_{x}\right)$ is $m_{1}$-integrable, and we define $m^{*} E$ for such an $E$ to be $\int m^{\prime}\left(E_{x}\right) d m_{1}$. Of course we don't know at this stage that there are any such sets $E$ or that, if there are, they form a $\sigma$-field. However we prove that every compact subset of $G$ is of this kind, then that all sets of the field (see definition below) generated by the compact sets are of this kind, then that all sets of the $\sigma$-field generated by the compact sets are of this kind. Then, after observing that $m^{*}$ is left invariant, we conclude that for these sets $m E=k m^{*} E$, which is the desired result. 
Throughout this paper $G, G^{\prime}$ and $G_{1}$, and $m, m^{\prime}$ and $m_{1}$, will have the meanings stated in Theorem 1 . We shall also refer to the topologies of $G, G^{\prime}$ and $G_{1}$, which we denote respectively by $T, T^{\prime}$ and $T_{1}$. We shall consider the $T^{\prime}$-topology not only on $G^{\prime}$ but on all of $G$-that is, the topology on $G$ in which the neighborhoods of $e$ are the $T^{\prime}$-open subsets of $G^{\prime}$ will be the $T^{\prime}$-topology on $G$. We follow Weil in using the notation $L_{+}(G)[5$, p. 32] for the continuous nonnegative functions which vanish outside a $T$-compact set, and similarly use the notation $L_{+}\left(G^{\prime}\right)$ and $L_{+}\left(G_{1}\right)$ in connection with $G^{\prime}$ and $G_{1}$.

Definition. Let $A$ be the class of those functions $f(x)$ on $G$ which are $m^{\prime}$-integrable on every coset of $G^{\prime}$ in $G$, that is, for each $x, f\left(x x^{\prime}\right)$ is an integrable function of $x^{\prime}$. Then for each $f \in A$ the function $\int f\left(x x^{\prime}\right) d m^{\prime}$ is constant on the cosets of $G^{\prime}$ and hence defines a function $f_{1}\left(x_{1}\right)$ on $G_{1}$. We denote the mapping which takes each $f \in A$ into its corresponding $f_{1}$ by $\Phi$.

Definition. Let $B$ be the collection of those subsets $E$ of $G$ for which both of the following are true:

(1) For every $x_{1}$ the intersection $E_{x_{1}}$ of $E$ with the coset $x_{1}$ is $m^{\prime}$-measurable, that is, $E_{x_{1}}=E \cap x G^{\prime}$ is $m^{\prime}$-measurable for every $x \in G$.

(2) The function $f\left(x_{1}\right)=m_{1}\left(E_{x_{1}}\right)$ is $m_{1}$-integrable with a finite $m_{1}$-integral.

This definition could be restated as follows: $B$ is the collection of sets whose characteristic function $f$ is in $A$ and such that $\Phi f$ is $m_{1}$-integrable.

We now break the proof of Theorem 1 into a succession of theorems and then break the proof of each of these into a number of lemmas.

Theorem 2. Every T-compact subset of $G$ is in $B$.

Lemma 1. If $f \in L_{+}(G)$ then $f \in A$ and $\Phi f \in L_{+}\left(G_{1}\right)$.

Proof. Because $G^{\prime}$ is closed in $G$ and the $T^{\prime}$-topology is that induced on $G^{\prime}$ by the $T$-topology on $G$ it is immediate that $f\left(x x^{\prime}\right)$ is in $L_{+}\left(G^{\prime}\right)$ for each $x \in G$, and hence $f \in A$.

Let $\phi$ be the natural homomorphism of $G$ onto $G_{1}$ and $C$ a compact subset of $G$ outside of which $f$ vanishes. Then clearly $f_{1}=\Phi f$ vanishes outside $\phi(C)$. Because $f$ is uniformly continuous on $G$ it follows that $\int f\left(x x^{\prime}\right) d m^{\prime}$ is continuous on $G$, and hence that $f_{1}$ is continuous on $G_{1}$.

Lemma 2. If $C$ is a $T$-compact set with characteristic function $f$ then $f \in A$ and $\Phi f$ is a bounded $x_{1}$-function which vanishes outside some $T_{1}$-compact set.

Proof. Clearly $f \in A$ because $C \cap x G^{\prime}$ is $T^{\prime}$-compact, hence $m^{\prime}$-measurable. Choose any $g \in L_{+}(G)$ such that $g \geqq f$. Then $\Phi g \geqq \Phi f$, and since $\Phi g \in L_{+}\left(G_{1}\right)$, by Lemma 1, this lemma follows.

To finish the proof of Theorem 2 we must show that $\Phi f$ ( $f$ the characteristic function of a $T$-compact $C$ ) is $m_{1}$-measurable. This is the purpose of Lemmas 3 and 4.

Lemma 3. If $C$ is any compact subset of $G$ with $m C=a$, and $b>a$, then there 
exists an open set $O$ such that every $m$-measurable set $E$ with $m E \geqq b$ contains $a$ point outside $C \bar{O}$.

Proof. We first prove that inf $m(C O)=m C$, where this inf is taken over all open subsets of $G$ which are contained in a compact subset-for such $O$ the set $C O$ is of course measurable. To prove this we assume the opposite, that this inf equals $c>m C$. Then choose a sequence $\left\{O_{n}\right\}$ of open sets such that $m\left(C O_{n}\right) \downarrow c$, and let $F=\cap O_{n}$, so that $F \supseteq C$ and $m F=c>m C$. Choose a compact set $D$ in $F-C$ of positive measure. Because $C \| D$ we can find an open $O$ such that $C O \| D$, hence $m\left(C O_{n}\right) \geqq m\left(C\left(O \cap O_{n}\right)\right)+m D$, contradicting the choice of the sequence $\left\{O_{n}\right\}$.

To prove the lemma, let $O$ be any open subset of $G$, whose closure $\bar{O}$ is compact and such that $m(C \bar{O})<b$. The previous paragraph shows that such an $O$ exists. Clearly $m E \geqq b$ implies that $E$ contains a point outside $C \bar{O}$.

Lemma 4. If $C$ is $T$-compact and $c$ is any positive real number then

$$
D=\left[x \mid x \in C \text { and } m^{\prime}\left(C \cap x G^{\prime}\right) \geqq c\right]
$$

is T-compact.

Proof. To show $D$ is $T$-compact it is sufficient to show it is $T$-closed, since $D \subseteq C$; we shall suppose that $D$ is not closed and obtain a contradiction. Suppose then that $z \in D$ but every $T$-neighborhood $N$ of $z$ intersects $D$. For each such $N$ we choose a point $z_{N} \in N \cap D$. We now consider, for each $N$, the group translation $T_{N}$ defined by $T_{N} x=z z_{N}^{-1} x$. Clearly $T_{N}$ carries the coset containing $z_{N}$ into the coset containing $z$. Hence $m^{\prime}\left(\left(T_{N} C\right) \cap z G^{\prime}\right) \geqq c$ for every $N$ while $m^{\prime}\left(C \cap z G^{\prime}\right)<c$. Applying the preceding lemma, with the $T^{\prime}$-topology and $m^{\prime}$ measure, we find a $T^{\prime}$-open set $O^{\prime}$, and then for each $N$ a point $y_{N}$ such that $y_{N} \in\left(T_{N} C\right) \cap z G^{\prime}$ but $y_{N} \notin\left(C \cap z G^{\prime}\right) O^{\prime}$. Now let $w_{N}=T_{N}^{-1} y_{N}=z_{N} z^{-1} y_{N}$.

Now let $w$ be a point with the following property: for every neighborhood of $w$ and every $N$ there are infinitely many $w_{M}$, with $\mathrm{M} \subseteq N$, in this neighborhood of $w$. By standard compactness arguments it is clear that such a $w$ exists. Our contradiction will consist in showing that $w$ both belongs and does not belong to $C \cap z G^{\prime}$. Because the $w_{N} \in C$ it follows that $w \in C$, and because $w_{N}=z_{N} z^{-1} y_{N}$ it follows from the way the $z_{N}$ were chosen that $w$ also has the property: for every neighborhood of $w$ and every $N$ there are infinitely many $y_{M}$, with $M \subseteq N$, in this neighborhood of $w$. Since the $y_{N}$ all belong to $z G^{\prime}$ and $z G^{\prime}$ is $T$-closed it follows that $w \in z G^{\prime}$; hence $w \in C \cap z G^{\prime}$. However, because the $Y_{N} \notin\left(C \cap z G^{\prime}\right) O^{\prime}$ and because the $T^{\prime}$-topology is that induced from the $T$-topology it follows that $w \notin C \cap z G^{\prime}$. This is the desired contradiction.

Proof of Theorem 2. Let $C$ be a $T$-compact subset of $G$ and $f$ its characteristic function. By Lemma $2, f \in A$, and $\Phi f$ is a bounded function vanishing outside a $T_{1}$-compact set. Lemma 4 then implies that for each $c>0$ the set $\left[x_{1} \mid f_{1}\left(x_{1}\right) \geqq c\right]$ is $T_{1}$-compact, since this set is just the image of $D=[x \mid x \in C$ 
and $\left.m^{\prime}\left(C \cap x G^{\prime}\right) \geqq c\right]$ under the natural homomorphism of $G$ onto $G_{1}$. This implies the $m_{1}$-measurability of $f_{1}=\Phi f$. Hence $C \in B$.

Definition. A field of sets is a family of sets closed under the operations of difference and finite union, that is, if $E, F$ belong to the family then $E-F$ and $E \cup F$ also belong to the family. We denote the field generated by the $T$-compact sets by $K$ (that is, $K$ is the smallest field containing all compact subsets of $G$ ).

The next two lemmas are for the purpose of proving that $K \subseteq B$.

Lemma 5. Let the sets $C_{1}-D_{1}, \cdots, C_{n}-D_{n}$ be mutually disjoint, where the $C_{i}$ and $D_{i}$ are compact, and let $C$ and $D$ be compact. Then the set $\left(C_{1}-D_{1}\right) \cup \ldots$ $\cup\left(C_{n}-D_{n}\right) \cup(C-D)$ is expressible as a union $\cup_{j}\left(C_{j}^{\prime}-D_{j}^{\prime}\right)$ with the sets $C_{i}^{\prime}-D_{i}^{\prime}$ mutually disjoint, and where the $C_{i}^{\prime}$ and $D_{i}^{\prime}$ are compact.

Proof. A direct calculation shows that $\left(C_{1}-D_{1}\right) \cup \cdots \cup\left(C_{n}-D_{n}\right)$ $\cup(C-D)$ is expressible in the following form, and that the terms are mutually disjoint,

$$
\begin{aligned}
\cup_{i}\left[C_{i}-\right. & \left.\left(C_{i} \cup D_{i}\right)\right] \cup \cup_{i}\left[\left(C_{i} \cap C\right)-D_{i}\right] \\
& \cup \cup_{I}\left[\left(D_{i_{1}} \cap \cdots \cap D_{i_{k}} \cap C\right)-\left(C_{i_{1}} \cup \cdots \cup C_{i_{n-k}} \cup D\right)\right],
\end{aligned}
$$

where the last union is over all subsets $I=\left(i_{1}, \cdots, i_{n}\right)$ of the integers $1, \cdots, n$, the integers $j_{1}, \cdots, j_{n-k}$ being the complementary set of $I$.

LEMMA 6. $K$ consists of precisely those sets of the form $U_{i}\left(C_{i}-D_{i}\right)$, where the $C_{i}$ and $D_{i}$ are compact, and the $C_{i}-D_{i}$ are mutually disjoint.

Proof. Clearly all such sets lie in $K$. To show the converse we note that repeated application of Lemma 5 shows that a finite union of sets of this form is again of this form. If we use this and the fact that $\left(C_{1}-D_{1}\right)$ $-\left(C_{2}-D_{2}\right)=\left[\left(C_{1} \cap D_{2}\right)-D_{1}\right] \cup\left[C_{1}-\left(D_{1} \cup C_{2}\right)\right]$ it is trivial that the difference of two sets of this form is again of this form. Hence the lemma is proved.

THEOREM 3. $K \subseteq B$, that is, every set in the field generated by the $T$-compact sets is in $B$.

Proof. It is trivial that if $E_{1}, \cdots, E_{n}$ are in $B$ and the $E_{i}$ are mutually disjoint, then $\cup_{i} E_{i} \in B$, and that if $E_{1}, E_{2}$ are in $B$ and $E_{1} \supseteq E_{2}$ then $E_{1}-E_{2} \in B$. If $E \in K$ then write $E=\cup_{i}\left(C_{i}-D_{i}\right)$, where this is a disjoint union and the $C_{i}, D_{i}$ are compact. Replacing $C_{i}$ by $C_{i}^{\prime}=C_{i} \cup D_{i}$, if necessary, we can even make sure that $C_{i} \supseteq D_{i}$. Since we know by Theorem 2 that the $C_{i}$ and $D_{i}$ are in $B$ it follows that $E \in B$, which proves Theorem 3.

Lemma 7. Let $\Sigma$ be the $\sigma$-field generated by the T-compact sets. Then if $E \in \Sigma$, the characteristic function $f$ of $E$ is in $A$, and $\Phi f$ is $m_{1}$-measurable.

Proof. We use the theorem of Saks which states that the "normal class" generated by a field of sets is the same as the $\sigma$-field generated by the field 
[4, p. 85, statement (9.7)]. A "normal class" is a family of sets closed under the operations of disjoint countable union and limit of decreasing sequences. Saks states his theorem not for a $\sigma$-field but for something slightly different, but the proof proves the theorem in the form we have stated it.

Since we know the conclusion of the lemma in case $E \in K$ and since the family of those sets $E$ such that $f \in A$ and $\Phi f$ is $m_{1}$-measurable clearly form a normal class the lemma follows from the theorem of Saks.

Definition. For each $E \in \Sigma$ we define the set function $m^{*} E$ by

$$
m^{*} E=\int \Phi f d m_{1},
$$

where $f$ is the characteristic function of $E$.

Proof of Theorem 1. To prove Theorem 1 we need to know enough about $m^{*} E$ to apply the uniqueness theorem for Haar measure. Clearly $m^{*} E$ is a measure, if we complete this set function, and by Theorem 2 it is finite for compact sets. If we can show that $m^{*}$ is left invariant then the uniqueness of Haar measure will immediately yield Theorem 1 .

If $E$ is any set in $\Sigma$ with characteristic function $f(y)$ then the characteristic function of $x E$ is $g(y)=f\left(x^{-1} y\right)$. Then it is trivial that $\Phi f$ is a left translation of $\Phi g$, hence $\int \Phi f d m_{1}=\int \Phi g d m_{1}$, so that $m^{*} E=m^{*}(x E)$. Thus Theorem 1 is proved.

\section{BIBLIOGRAPHY}

1. W. Ambrose, P. Halmos, and S. Kakutani, Duke Math. J. vol. 9 (1942) pp. 43-47.

2. P. Halmos, Duke Math. J. vol. 8 (1941) pp. 386-392.

3. H. Cartan, Bull. Sci. Math. (2) vol. 66 (1942) pp. 236-245.

4. S. Saks, Theory of the integral, $2 \mathrm{~d}$ rev. ed., Warsaw, 1937.

5. A. Weil, L'Integration dans les groupes topologiques et ses applications, Actualités Scientifiques et Industrielles, no. 869, Paris, 1940.

UNIVERSITY OF MichigaN, AnN Arbor, Mich. 